\title{
Molecularly Imprinted Polymer Applied to the Solid Phase Extraction for Trace Analysis of Tribenuron- Methyl in Water and Soil Samples
}

\author{
Mingming Zhang and Junping Wang* \\ Key Laboratory of Food Nutrition and Safety, Tianjin University of Science \& Technology, Tianjin, China \\ *Corresponding author
}

\begin{abstract}
A molecularly imprinted polymer (MIP) was synthesized by bulk polymerization method using tribenuronmethyl (TBM) as the template molecule, methacrylic acid (MAA) as the functional monomer, ethylene glycol dimethacrylate (EDMA) as the cross-linker and $2,2^{\prime}$-azobisisobutyronitrile (AIBN) as the initiator. After the polymerization, the template molecule was removed from the polymer by an accelerated solvent extractor (ASE). The resulted MIP was evaluated by static absorption, kinetic absorption and selective experiments with high performance liquid chromatography (HPLC). The polymer was further used as the solid phase extraction (SPE) material to determine trace TBM coupled with HPLC, as a result, the recoveries of the method were from 90.18 to $92.69 \%$ and the RSD\% were less than 2.52 in the water and soil samples. Low detection limit was also obtained in the method evaluation study.
\end{abstract}

Keywords-molecularly imprinted polymer; tribenuron-methyl; solid phase extraction; high performance liquid chromatography

\section{INTRODUCTION}

Sulfonylureas herbicides (SUs) were first introduced in 1982 by DuPont Agricultural Products [1]. Tribenuron-methyl (TBM) is one of the SUs, which have been developed into a largest herbicide used for crops protection due to the fact that they have the characteristics of low dosage, low mammalian toxicity and easy degradation to the innocuous compounds after application.

In recent years, pesticide have become the focus of international trade dispute. To overcome the problem of international trade dispute, the implementation of effiective SUs pesticide residues management and food safety ensurance is essential, which required the development of fast and accurate examination method of SUs pesticide residues.

Now, several analytical techniques, including highperformance liquid chromatography (HPLC) [2,4,8], high performance liquid chromatography-mass spectrometry (HPLC-MS) [3-6], capillary electrophoresis with ultraviolet detector (CE-UV) [7], and immunoassay [9], have been used to determine trace SUs in different samples. In spite of using any techniques, sample pretreatment should not be neglected especially when examined the trace level organic contaminants in complex matrices. Among the lots of sample preparation techniques, solid-phase extraction (SPE) is routinely used for the extraction of compounds from liquid or solid matrices. But it is difficult to elute analyte due to low content and complex matrix, which required more sufficient selectivity for SPE sorbents [10].

Molecularly imprinted polymers (MIPs) are artificial materials with highly specific recognition ability for target molecules [11]. In the polymer synthesis stage, a functional monomer forms a complex with the template molecule, which is being removed by extensive washing steps after polymerization. Consequently, the template leaves its cavity in the polymer structure, which is responsible for the recognition properties of the MIPs [12]. Because of their molecular recognition abilities, MIPs were extensively used in many fields, such as biochemical sensors [13], simulating enzymecatalyzed reactions [14], drug delivery systems [15], and SPE [10, 16-19].

In this study, the MIP of TBM was synthesized by bulk polymerization method. Binding characteristics and selectivity of the MIP has been evaluated through static absorption, kinetic absorption and selective experiments. The polymer was applied as sorbent of SPE to determine trace TBM in water and soil samples by HPLC.

\section{MATERIALS AND METHOD}

\section{A. Materials and Reagents}

TBM, Thifensulfuron-methyl (TFSM), Metsulfuron-methyl (MSM), Ethametsulfuron-methyl (EMSM) (Figure 1) and ethylene glycol dimethacrylate (EDMA) were obtained from Fluka (Sigma-Aldrich, USA). Methacrylic acid (MAA), 2,2'Azobisisobutyronitrile (AIBN) and toluene were purchased from Tianjin Chemical Reagent Factory (Tianjin China). MAA and AIBN were purified before using. Acetonitrile and other chemicals were supplied by concord Co., (Tianjin, China). Doubly deionized water (DDW, $18 \mathrm{MXN} \mathrm{cm}$ ) obtained from a WaterPro water system (Labconco Corp, Kansas City, MO) was used throughout the experiments. The water samples were collected from Guangzhou village and tap water from our lab, respectively, and soil samples were obtained from Tianjin village.

\section{B. Instrumentation}

HPLC system is consisted of two LC-20ATvp pumps and a Shimadzu SPD-20AVP ultraviolet detector (Shimadzu, Kyoto, Japan). All separations were achieved on a reversed-phase column (Thermo-C18, $5 \mu \mathrm{m}, 4.6 \mathrm{~mm} \times 250 \mathrm{~mm}$, Thermo, USA) at a mobile phase flow rate of $1.0 \mathrm{~mL} \mathrm{~min}{ }^{-1}$ under isocratic conditions at $30^{\circ} \mathrm{C}$ of column temperature. Class-vp 
software was used to acquire and process spectral and chromatographic data. The mobile phase was consisted of $0.01 \%$ trifluoroacetic acid solution as mobile phase $\mathrm{A}$ and acetonitrile as mobile phase B with UV detection at $230 \mathrm{~nm}$. The $0.01 \%$ trifluoroacetic acid solution and acetonitrile were filtered through $0.22-\mu \mathrm{m}$ microfiber filters of medium pore diameter (Phenomenon, Tianjin, China) prior to use. An ASE300 accelerated solvent extractor (Dionex Corporation, USA) was used to remove template molecule.

\section{Synthesis of MIP}

The MIP was prepared by bulk polymerization. Briefly, $395.4 \mathrm{mg}$ of TBM $(1.0 \mathrm{mmol})$ was dissolved in $4.0 \mathrm{~mL}$ of $\mathrm{N}, \mathrm{N}$-dimethylformamide (DMF) and $2.0 \mathrm{~mL}$ of toluene, and mixed with $0.278 \mathrm{~mL}(3.0 \mathrm{mmol})$ of MAA. The mixture was left reaction for $2 \mathrm{~h} .1 .32 \mathrm{~mL}(7.0 \mathrm{mmol})$ of cross-linker EDMA and $0.06 \mathrm{~g}$ of initiator AIBN were added. After ultrasonicated and purged with nitrogen, the mixture was sealed and thermally initiated in a water bath at $60^{\circ} \mathrm{C}$ for $24 \mathrm{~h}$. After the polymerization, the rigid polymer was crushed, and sieved through a 200 mesh steel sieve. The obtained polymer was washed with acetonitrile by ASE three times to extract TBM. The extraction conditions were as follows: 5 min heatup, 8 min static period, $100 \mathrm{sec}$ purge, 5 cycles. All extraction procedures were carried out at pressure up to $1500 \mathrm{psi}$ and the temperature was set at $80^{\circ} \mathrm{C}$. The product was dried in a vacuum drying oven at $80^{\circ} \mathrm{C}$ for $12 \mathrm{~h}$.

For comparison, a non-imprinted polymer (NIP) was also prepared in the same way except the absence of template.

\section{MIP Binding Characteristics and Selectivity Evaluation}

\section{1) Adsorption capacity study}

To measure adsorption capacity, $50.0 \mathrm{mg}$ of MIP was mixed with $10.0 \mathrm{~mL}$ of methanol solution containing TBM at different concentrations $\left(20.0 \mathrm{mg} \mathrm{L}^{-1}, 50.0 \mathrm{mg} \mathrm{L}^{-1}, 80.0 \mathrm{mg}\right.$ $\left.\mathrm{L}^{-1}, 100.0 \mathrm{mg} \mathrm{L}^{-1}, 120.0 \mathrm{mg} \mathrm{L}^{-1}\right)$. The mixtures were shaken $\left(160\right.$ times min $^{-1}$ ) for $10 \mathrm{~h}$ with a horizontal shaker and then separated centrifugally (4000 rpm) for $5 \mathrm{~min}$. The supernatant was determined for the unbound TBM by HPLC. The same procedure was applied to test the static absorption of the NIP.

\section{2) Adsorption kinetic study}

$50.0 \mathrm{mg}$ of the MIP sorbent was mixed with $10.0 \mathrm{~mL}$ of $20.0 \mathrm{mg} \mathrm{L}^{-1}$ methanol solution of TBM. The mixture was shaken at different times $(2 \mathrm{~h}, 4 \mathrm{~h}, 6 \mathrm{~h}, 8 \mathrm{~h}, 10 \mathrm{~h}$ and $14 \mathrm{~h})$ and then separated centrifugally $(4000 \mathrm{rpm})$ for $5 \mathrm{~min}$. The unbound TBM in the supernatant was determined by HPLC.

\section{3) Selectivity study}

Competitive recognition studies were performed with TBM, TFSM, MSM and EMSM. The MIP (50.0 mg) was added to a flask containing $10.0 \mathrm{~mL}$ of $20.0 \mathrm{mg} \mathrm{L}^{-1}$ mixed methanol solution of TBM, TFSM, MSM and EMSM, shaken for $10 \mathrm{~h}$ and then centrifugally separated. The concentration of the supernatant was determined by HPLC.
E. Procedures for Selective SPE Coupled with HPLC (SPEHPLC) Determination of Trace TBM Using MIP as SPE Material

\section{1) Preparation of water samples}

The water sample $(250.0 \mathrm{~mL})$, determined to be free of the analyte, was spiked with TBM methanol solution, adjusted to $\mathrm{pH} 5.2$

\section{2) Preparation of soil samples}

The free soil sample $(5.0 \mathrm{~g})$ and $10.0 \mathrm{~mL}$ of TBM methanol solution was spiked and mixed with $50.0 \mathrm{~mL}$ of $0.2 \mathrm{~mol} \mathrm{~L}^{-1}$ ammonia water/methanol $(1: 1, \mathrm{v} / \mathrm{v})$ extraction solution. After being shaken (180 times min $\left.^{-1}\right)$ with a horizontal shaker for $1 \mathrm{~h}$, the mixture was centrifuged at $1500 \mathrm{~g}$ for $10 \mathrm{~min}$. The supernatant was diluted with DDW to $250.0 \mathrm{~mL}$ and adjusted to $\mathrm{pH} 5.2$

\section{3) SPE procedure}

To evaluate the applicability of the MIP as sorbent of SPE, a column packed with $50.0 \mathrm{mg}$ of MIP was equilibrated with $5.0 \mathrm{~mL}$ of acetonitrile and $10.0 \mathrm{~mL}$ DDW, successively. Prepared water or soil samples was pumped into the SPE column to enrich the analyte. Then the column was eluted with $7.0 \mathrm{~mL}$ of a mixture of acetonitrile/acetic acid $(95 / 5, \mathrm{v} / \mathrm{v})$. The eluate was evaporated to dryness under a stream of dry nitrogen. The residue was redissolved with $1.0 \mathrm{~mL}$ of acetonitrile and determined by HPLC.

\section{RESUlTS AND DISCUSSION}

\section{A. MIP Synthesis}

The bulk polymerization method is a simpler and more convenient technique for preparing MIP. In the present study, MAA was selected as the functional monomer and EDMA was selected as the cross-linker. When the molar ratio of template molecule, functional monomer and cross-linker was 1:3:7, the polymer has the best adsorption property.

\section{B. Removal of Template from MIP}

After the polymerzation, removal of the template from the polymer before being used is of great importance in preparation procedure. The template bleeding can directly influence the detection limit and precision, even giving erroneous results if the MIP is applied to solid-phase extraction with incomplete removal of template [20]. Compared with three removing template methods of soxhlet extraction (SE), ultrasonic assisted extraction (USE) and ASE, the experimental results showed that ASE was the more effective method for rapid removal of template from MIP. ASE can elevate the efficiency of extraction through the action crippled between analyte and matrix and activation energy of desorption decreased at high temperatures and pressures [21]. In the present experiment, the polymers processed by ASE obtained a larger capacity $\left(1.06 \mathrm{mg} \mathrm{g}^{-1}\right)$ than SE $\left(0.95 \mathrm{mg} \mathrm{g}^{-1}\right)$ and USE $\left(0.57 \mathrm{mg} \mathrm{g}^{-1}\right)$. 


\section{Evaluation of MIP}

\section{1) Evaluation of adsorptive property}

The adsorption capacity was evaluated by static adsorption experiment. The result suggested that the MIP has higher adsorption capacity $\left(1.06 \mathrm{mg} \mathrm{g}^{-1}\right)$ than that of the NIP $(0.71 \mathrm{mg}$ $\left.\mathrm{g}^{-1}\right)$ at the same concentration of TBM. The adsorption capacity of the MIP for TBM was increased with increasing the initial concentration of TBM, and reached saturation at the $100.0 \mathrm{mg} \mathrm{L}^{-1}$ concentration of TBM (Figure 2).

The adsorption kinetic test of TBM by the MIP was also examined. It could be seen from Figure 3 that $95.6 \%$ of TBM could be adsorbed by the MIP after adsorbing for $8 \mathrm{~h}$, and adsorption equilibrium was obtained within $10 \mathrm{~h}$. If the concentration of TBM was lower, the time to saturation would become shorter. The prepared MIP adsorption rate was obviously fast compared with general bulk polymerization method, which reached adsorption equilibrium need $24 \mathrm{~h}$ [22].

\section{2) Selectivity of the MIP}

Competitive adsorption test of MIP and NIP towards TBM, TFSM, MSM and EMSM were performed and the results were shown in Table 1.

The distribution coefficient (kd) of MIP for TFSM, MSM, EMSM and TBM were 4.76, 7.62, 17.10 and 66.9, respectively, it demonstrated that the uptake capacity of MIP for TBM was higher than that of the other three SUs. In contrast the kd of NIP for TFSM, MSM, EMSM and TBM were 2.61, 2.80, 5.77 and 5.31, respectively. The selectivity coefficients $(\mathrm{k})$ for the three SUs were 14.05, 8.78 and 3.91 for MIP, and 2.03, 1.90 and 0.92 for NIP. These results indicated that the synthesized MIP could recognize the template specifically. Figure 4 also illustrated that MIP had specific absorption to TBM. These results showed that the imprinting process was successful and the specific binding sites have been formed within the MIP during the imprinting process.

\section{Optimization of SPE Condition for the Determination of TBM}

In this experiment, We optimized several key parameters which influenced the efficiency of SPE such as sample acidity, eluent, and its concentration and volume. $250.0 \mathrm{~mL}$ of DDW used for optimizing the SPE process were spiked $1 \mu \mathrm{g} \mathrm{L} \mathrm{L}^{-1}$ TBM. The influence of sample $\mathrm{pH}$ was investigated in the $\mathrm{pH}$ range of 3.0-7.0. The results showed that the chromatography peak areas of TBM were maximal range from 5.0 to 5.4, which meant that the retention of TBM on the SPE was obviously influenced by the $\mathrm{pH}$ of the sample solution. Out of the optimum $\mathrm{pH}$ range, the chromatographic peak area of TBM decreased. Therefore, TBM could be effectively absorbed by the SPE in the $\mathrm{pH}$ range of 5.0-5.4. The $\mathrm{pH}=5.2$ was chosen as the experimental condition. Different solvents were tested to identify their influence on the elution of TBM from the SPE. A better result was obtained when using acetonitrile as the eluent compared with methanol, dichloromethane and DMF. It was known that the template molecules could bind to the MIP by hydrophobic interaction, so acetic acid could weak hydrogen bonding force and increase the eluting strength. The concentration of acetic acid in the eluent was studied in the range of 1 to $10 \%$. As the concentration of acetic acid was up to $5 \%$, the elution efficiency had no obvious change. Finally, a mixture of acetonitrile/acetic acid $(95: 5, \mathrm{v} / \mathrm{v})$ was selected as the elution with a high recovery. The effect of elution volume on the elution efficiency was tested. When the volume of elution reached $7.0 \mathrm{~mL}, 98 \%$ of the TBM could be eluted.

\section{E. Analysis of Real Samples Using SPE-HPLC}

The analytical figures of merit of the SPE-HPLC method for the determination of trace TBM was evaluated under optimal experimental conditions. Linearity was achieved at the concentration range between 0.1 and $30 \mu \mathrm{g} \mathrm{L^{-1 }}$ in TBM aqueous solution through the SPE procedure with a good correlation coefficients $(\mathrm{r} 2=0.9912), \mathrm{A}($ peak area $)=44104 \mathrm{C}$ (concentration in $\mu \mathrm{g} \mathrm{L}^{-1}$ )-1646.5.

To validate the usefulness of the SPE-HPLC method for the determination of trace TBM, three real samples, Guangzhou village water, tap water and soil samples were analyzed under optimal experimental conditions. The detection limits $(\mathrm{S} / \mathrm{N}=3)$ were $0.1 \mu \mathrm{g} \mathrm{L}^{-1}, 0.1 \mu \mathrm{g} \mathrm{L}^{-1}$ and $2.0 \mu \mathrm{g} \mathrm{kg}^{-1}$ for Guangzhou village water, tap water and soil sample, respectively. Good recoveries (Table 2) were obtained between $90.18 \%$ and $92.69 \%$ at spiked concentrations with $\mathrm{RSD} \%$ ranged from 0.99 to $2.52(n=3)$. Typical chromatograms of water and soil samples were shown in Figure 5. It demonstrated that the synthesized sorbent could be used for the determination of trace TBM in the real samples and suggested potential practical application.

\section{CONCLUSION}

A TBM MIP was successfully synthesized by bulk polymerization method. The prepared material showed high adsorption capacity, affinity and selectivity towards the template TBM. It has been used for the determination of trace TBM from water and soil samples by SPE-HPLC. Good recoveries at low concentration levels and low limit of detection were obtained through the established method in this paper.

\section{ACKNOWLEDGEMENTS}

This work was supported by the Ministry of Science and Technology of China (No. 2008AA10Z420) and the National Natural Science Foundation of China (No. 20775054 and No. 30872126).

\section{REFERENCES}

[1] AYANO Eri, KANAZAWA Hideko, ANDO Masanori, and NISHIMURA Tetsuji, Anal. Chim. Acta 507, 211 (2004).

[2] Jing-fu Liu, Jing-bo Chao, and Gui-bin Jiang, J. Chromatogr. A 995, 21 (2003).

[3] R. Carabias-Martínez, E. Rodríguez-Gonzalo, E. Herrero-Hernández, and J. Hernández-Méndez, Anal. Chim. Acta 517, 71 (2004)

[4] Eri Ayano, Hideko Kanazawa, Masanori Ando, and Tetsuji Nishimura, Anal. Chim. Acta 507, 211 (2004).

[5] S. Polati, M. Bottaro, P. Frascarolo, F. Gosetti, V. Gianotti, and M.C. Gennaro,J. Chim. Acta. 579146 (2006).

[6] Ye Guibiao, Zhang Wei, Cui Xin, Pan Canping, and Jiang Shuren, Chin. J. Anal. Chem. 34, 1207 (2006).

[7] Giovanni Dinelli, Alberto Vicari, and Vincenzo Brandolini, J. Chromatogr. A 700, 201 (1995). 
[8] LeEtta J. Marek, J. Agric. Food. Chem. 44, 3878 (1996).

[9] James F. Brady, JoLyn Turner, and David H. Skinner, J. Agric. Food. Chem. 43, 2542 (1995).

[10] Afshin Rajabi Khorrami and Shima Mehrseresht, J. Chromatogr. B 867, 264 (2008).

[11] F. Dickert, Anal. Bioanal. Chem. 389, 353 (2007).

[12] E. Caro, R. M. Marcé, F. Borrull, P. A. G. Cormack, and D. C. Sherrington, Trends Anal. Chem. 25, 143 (2006).

[13] Xiong Yan, Houjiang Zhou, Zhujun Zhang, Deyong He, and Chao He, J. Pharm. Biomed. Anal. 41, 694 (2006).

[14] Kurt Polborn and Kay Severin, Chem. Commun. 24, 2481 (1999).

[15] Carmen Alvarez-Lorenzo and Angel Concheiro, J. Chromatogr. B 804, 231 (2004).
[16] Qing-Zhi Zhu, Karsten Haupt, Dietmar Knoppa, and Reinhard Niessner, Anal. Chim. Acta 468, 217 (2002).

[17] Kaijie Tang, Shangwei Chen, Xiaohong Gu, Haijun Wang, Jun Daia, and Jian Tanga, Anal. Chim. Acta 614, 112 (2008).

[18] Xiangjun Liu, Zhiyong Chen, Rui Zhao, Dihua Shangguan, Guoquan Liu, and Yi Chen, Talanta 71, 1205 (2007).

[19] Xiangchao Dong, Ning Wang, Shengli Wang, Xuewei Zhang, and Zhijin Fan, J. Chromatogr. A 1057, 13 (2004).

[20] B. A. Rashid, R. J. Briggs, J. N. Hay, and D. Stevenson, Anal. Commun. 34, 303 (1997).

[21] Wu Peng-Ju, Yang Jun, Su Qing-De, Gao Yun, Zhu Xiao-Lan, and Cai Ji-Bao. Chin. J. Anal. Chem. 35, 484 (2007).

[22] Peng Tao, Du Xiaoyan, and Li Jun suo. Chin. J. Pest. Sci. 3, 1 (2001).

TABLE I. COMPETITIVE LOADING OF TBM, MSM, EMSM AND TFSM BY MIP AND NIP

\begin{tabular}{|c|c|c|c|c|c|c|c|c|c|c|}
\hline \multirow{2}{*}{ polymer } & \multicolumn{4}{|c|}{$k_{\mathrm{d}}^{\mathrm{a}}$} & \multicolumn{3}{|c|}{$k^{\mathrm{b}}$} & \multicolumn{3}{|c|}{$k^{, \mathrm{b}}$} \\
\hline & TFSM & MSM & EMSM & $T B M$ & TFSM & MSM & EMSM & TFSM & MSM & EMSM \\
\hline \multirow[t]{2}{*}{ MIP } & 4.76 & 7.62 & 17.1 & 66.9 & 14.05 & 8.78 & 3.91 & & & \\
\hline & & & & & & & & 6.92 & 4.62 & 4.25 \\
\hline NIP & 2.61 & 2.80 & 5.77 & 5.31 & 2.03 & 1.90 & 0.92 & & & \\
\hline
\end{tabular}

\footnotetext{
$\overline{{ }^{\mathrm{a}}} k_{\mathrm{d}}$, distribution coefficient; $k_{\mathrm{d}}=\left\{\left(C_{\mathrm{i}}-C_{\mathrm{f}}\right) / C_{\mathrm{f}}\right\} \times\{$ volume of solution $(\mathrm{mL})\} /\{$ mass of gel $(\mathrm{g})\}$, where $C_{\mathrm{i}}$ and $C_{\mathrm{f}}$ represent the initial and final concentrations

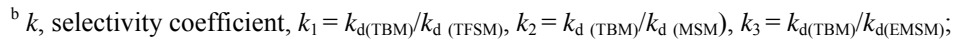

$k^{\prime}$, relative selectivity coefficient, $k^{\prime}=k_{\mathrm{MIP}} / k_{\mathrm{NIP}}$
}

TABLE II. ANALYTICAL RESULTS FOR THE DETERMINATION OF TRACE TBM IN REAL SAMPLES

\begin{tabular}{ccc}
\hline sample & spiked level $\left(\boldsymbol{\mu g} \mathbf{L}^{-\mathbf{1}}\right)$ & $\begin{array}{c}\text { recovery of TBM }(\%) \\
(\mathbf{m e a n} \pm \mathbf{R S D}, \mathbf{n}=\mathbf{3})\end{array}$ \\
\hline Guangzhou village water & 0.2 & $92.39 \pm 1.47$ \\
tap water & 0.2 & $92.55 \pm 2.52$ \\
soil sample & 4.0 & $90.18 \pm 0.99$ \\
& 10.0 & $92.69 \pm 1.40$ \\
\hline
\end{tabular}<smiles>COc1nc(C)nc(N(C)C(O)NS(=O)(=O)c2ccccc2C(=O)O)n1</smiles>

Triberusconmethy (TBM)<smiles>COC(=O)c1sccc1S(=O)(=O)NC(=O)Nc1nc(C)nc(OC)n1</smiles>

Thifenoulfuxon-methyl (TRSM)<smiles>COc1nc(C)nc(NC(O)C(=O)NC(=O)C2CC=CCC2C(=O)O)n1</smiles>

Metoulfuron methyl (MSM)<smiles>CCOc1nc(NC)nc(NC(=O)NS(=O)(=O)c2ccccc2C(=O)OC)n1</smiles>

Ethametoulfuron-methyl(EMSM)

FIGURE I. CHEMICAL STRUCTURES OF SUS USED IN THE COMPETITIVE ABSORPTION TEST 


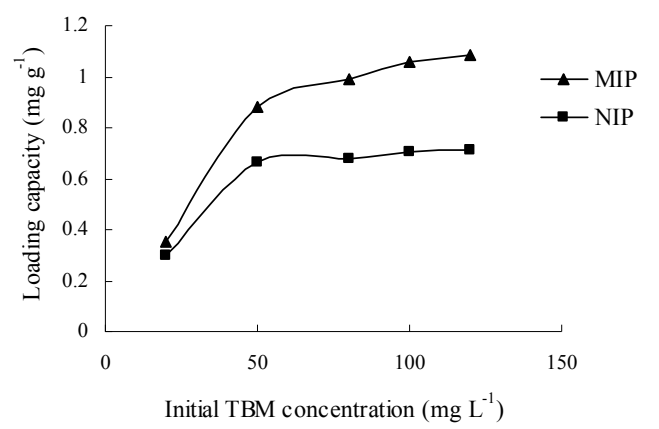

FIGURE II. ADSORPTION ISOTHERMS OF MIP AND NIP

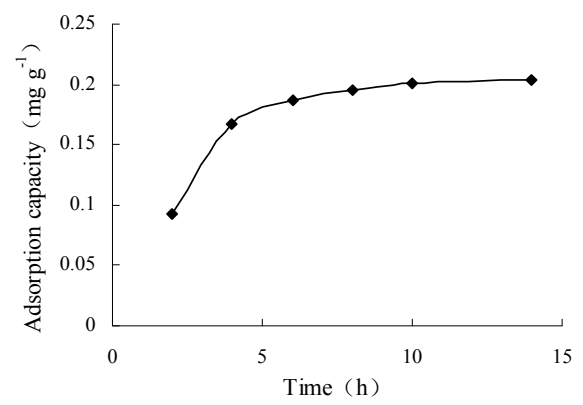

FIGURE III. STUDY OF KINETIC BINDING FOR 20 MG L-1 SMZ ONTO 50 MG OF MIP

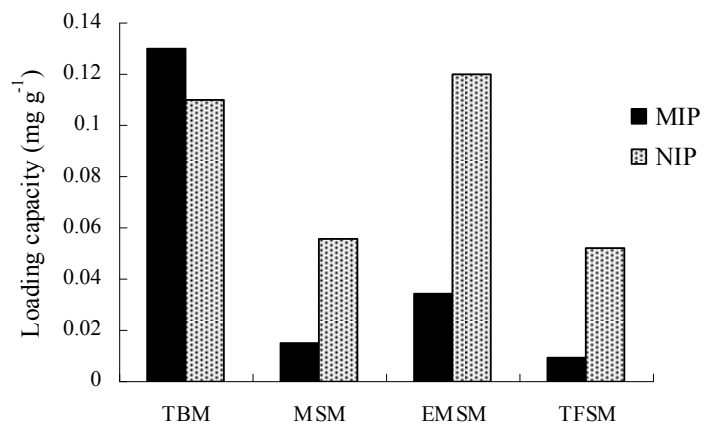

FIGURE IV. COMPETITIVE ADSORPTION OF TBM, MSM, EMSM AND TFSM USING MIP AND NIP

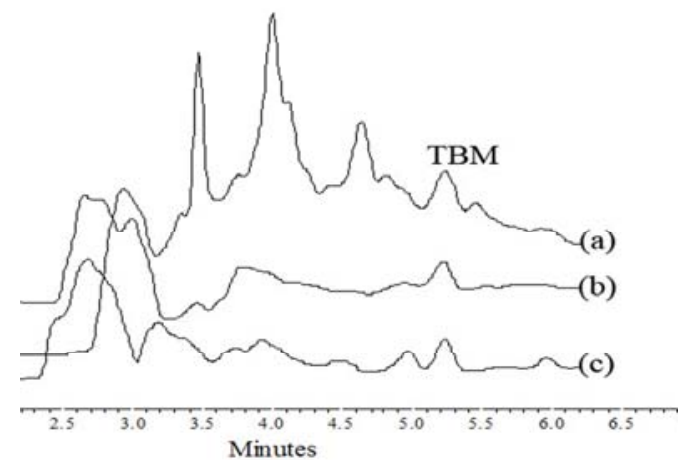

FIGURE V. CHROMATOGRAMS OBTAINED AT 230 NM FOR (A) 5.0 MG OF SOIL SAMPLE SPIKED WITH 10 MG KG-1 TBM (B) 250.0 ML OF TAP WATER SPIKED WITH 0.1 MG L-1 TBM (C) 250.0 ML OF GUANGZHOU VILLAGE WATER SPIKED WITH 0.1 MG L-1 TBM 